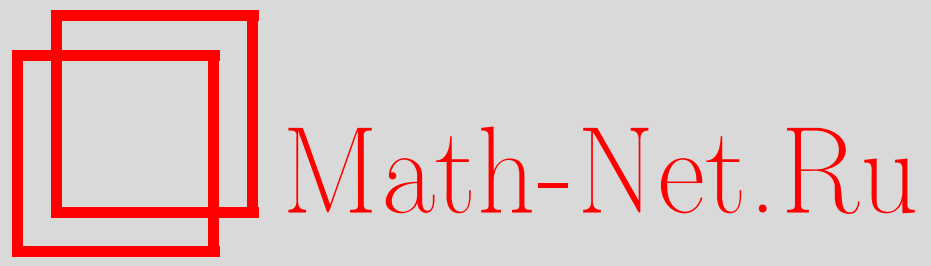

Ю. Ю. Кочетков, О геометрии одного класса плоских деревьев, Функи. анализ и его прил., 1999, том 33, выпуск 4, 78-81

DOI: https://doi.org/10.4213/faa385

Использование Общероссийского математического портала MathNet.Ru подразумевает, что вы прочитали и согласны с пользовательским соглашением

http://www. mathnet.ru/rus/agreement

Параметры загрузки:

IP: 3.85 .7 .115

26 апреля 2023 г., $14: 25: 26$

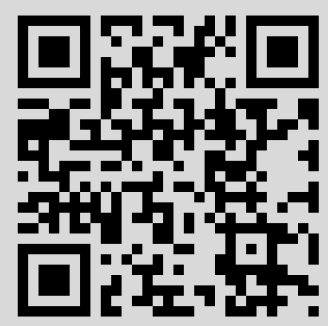


Чтобы имел место этот случай, необходимо и достаточно, чтобы выполнялись следующие два условия:

$$
\prod_{n=2}^{\infty} \frac{\alpha_{n}}{\mu_{n-1}}=\infty, \quad \prod_{n=1}^{\infty} \frac{\alpha_{n}}{\mu_{n}}=0 .
$$

Таким образом, доказана

Теорема 2. Пусть $A>0$ - вполне непрерывный оператор.

1 (случай (а)). Если набор чисел $\left\{\lambda_{n}\right\}_{n=1}^{\infty}$ таков, что $a_{k+1}<\lambda_{k}<a_{k}$, то этот набор является эллиптическими координатами некоторого вектора тогда и только тогда, когда $\prod_{n=1}^{\infty} \lambda_{n} / a_{n}>0$.

2 (случаи (b)-(d)). Если набор чисел $\left\{\lambda_{n}\right\}_{n=0}^{\infty}$ таков, что $\lambda_{0} \leqslant 0$ u $a_{k+1}<$ $\lambda_{k}<a_{k}$, то этот набор является эллиптическими координатами некоторого вектора тогда и только тогда, когда $\prod_{n=1}^{\infty} \lambda_{n} / a_{n}=0$.

В случае (а) для координат $x_{k}$ имеет место представление (7), а в случалх (b)-(d) - представление

$$
\left|x_{k}\right|^{2}=2\left(a_{k}-\lambda_{k-1}\right) \prod_{n \neq k} \frac{a_{k}-\lambda_{n-1}}{a_{k}-a_{n}} .
$$

ЛИТЕРАТУРА

1. Якоби К. Лекции по динамике. Гл. ред. общетехнич. лит., Л.-М., 1936. 2. $A p$ нольд В. И., Козлов В. В., Нейштадт А. И. Математические аспекты классической и небесной механики. Современные проблемы математики. Фундаментальные направления. Т. 3, Итоги науки и техники, ВИНИТИ, М., 1985. 3. Арнольд В. И. Математические методы классической механики. Наука, М., 1989. 4. Левин Б. Я. Распределение корней целых функций. ГИТТЛ, М., 1956.

Московский государственный университет

Поступило в редакцию им. М. В. Ломоносова,

11 мая 1999 г.

механико-математический факультет

УДК 519.1

\section{О геометрии одного класса плоских деревьев}

(C) 1999. Ю. Ю. Кочетков

Взаимно однозначное соответствие между классами изотопической эквивалентности плоских связных деревьев и классами линейной эквивалентности обобщенных многочленов Чебышёва позволяет определить истинную форму плоского связного дерева. В работе изучается истинная форма одного класса плоских деревьев - деревьев диаметра 4 с центральной валентностью 4.

Обобщенным многочленом Чебышёва называется многочлен $p(z) \in \mathbb{C}[z]$, имеющий два критических значения. Пусть $\alpha$ и $\beta$ - эти значения; тогда прообраз $p^{-1}([\alpha, \beta])$ отрезка $[\alpha, \beta]$ является плоским связным деревом $T(p)$ с $n=\operatorname{deg}(p)$ ребрами. Обратно, если $T$ - плоское связное дерево, то существует обобщенный многочлен Чебышёва $p$, такой, что $T(p)$ изотопно $T$ (т. е. существует сохраняющая ориентацию деформация плоскости, переводящая $T$ в $T(p)$ ). Такой многочлен мы будем называть обобщенным многочленом Чебышёва дерева $T$. 
Если $p$ и $q$ - обобщенные многочлены Чебышёва и $T(p)$ изотопно $T(q)$, то $p$ линейно эквивалентен $q$, т. е. $p(z)=a q(c z+d)+b, a, b, c, d \in \mathbb{C}[1]$. Таким образом каждое плоское связное дерево $T$ имеет истинную форму, т. е. форму (с точностью до вращений, сдвигов и растяжений), которую имеет дерево $T(p)$, изотопное $T$. Точных результатов об истинной форме мало. Представление о ней дает каталог [2], где приведены истинные формы всех деревьев с числом ребер, не превышающим 8. Отметим, что ребра, выходящие из одной вершины валентности $k$, делят полный угол на $k$ равных частей.

В данной работе изучается форма одного класса плоских деревьев. Введем необходимые определения. Мы рассматриваем деревья диаметра 4. Такое дерево имеет центральную вершину (центр) валентности $N$. Вершина, соединенная ребром с центром, называется боковой. Пусть $k_{1}, \ldots, k_{N}$ - валентности боковых вершин дерева $T$, перечисленные в порядке невозрастания; тогда мы говорим, что $T$ принадлежит типу $\left\langle k_{1}, \ldots, k_{N}\right\rangle$. Если $k_{i}$ попарно различны, то тип содержит $(N-1)$ ! попарно неизотопных деревьев. Если $T \in\left\langle k_{1}, \ldots, k_{N}\right\rangle$, то (с точностью до изотопии) $T$ определено циклическим порядком валентностей боковых вершин. Обобщенный многочлен Чебышёва $p$ дерева $T$ имеет степень $m=k_{1}+\cdots+k_{N}$. Если $\alpha$ и $\beta-$ его критические значения, то центр принадлежит множеству $p^{-1}(\alpha)$, а множество боковых вершин есть $p^{-1}(\beta)$. Пусть $\beta=0, a_{1}, \ldots, a_{N}$ - координаты боковых вершин, а центр находится в точке 0 . Тогда

$$
p(z)=\prod_{i=1}^{N}\left(z-a_{i}\right)^{k_{i}}=\sum_{i=0}^{m} b_{i} z^{i} .
$$

Так как 0 есть критическая точка порядка $N$, то $b_{1}=\cdots=b_{N-1}=0$, что дает нам систему

$$
\left\{\begin{array}{l}
k_{1} x_{1}+\cdots+k_{N} x_{N}=0, \\
k_{1} x_{1}^{2}+\cdots+k_{N} x_{N}^{2}=0, \\
\cdots \cdots \cdots \cdots \cdots \cdots \cdots \cdots \cdots \cdots+k_{N} x_{N}^{N-1}=0, \\
k_{1} x_{1}^{N-1}+\cdots+1
\end{array}\right.
$$

где переменные $x_{i}$ - это $1 / a_{i}$. Пусть $x_{N}=a_{N}=1$. Исключая $x_{1}, x_{2}, \ldots$, получим уравнение $F\left(x_{N-1}\right)=0$ степени $(N-1)$ !. Переменные $x_{1}, \ldots, x_{N-2}$ выражаются через $x_{N-1}$ полиномиально. Зная корень $F$, мы можем построить дерево (точнее множество боковых вершин). Углом между соседними боковыми вершинами валентностей $k_{i}$ и $k_{j}$ мы назовем угол между радиус-векторами $a_{i}$ и $a_{j}$ (т. е. аргумент отношения $a_{i} / a_{j}$ ). Величину $\left|a_{i}\right|$ будем называть модулем вершины валентности $k_{i}$. В данной работе будут доказаны некоторые утверждения о модулях и углах. Отметим, что параметры $k_{1}, \ldots, k_{N}$ в (1) можно считать положительными вещественными числами. В этом случае, однако, решение системы не имеет очевидного геометрического смысла.

ПримеР. Пусть $N=2$; тогда система (1) состоит из одного уравнения. Если $k<l$ - валентности боковых вершин, а $a, 1$ - их координаты, то $a=-k / l$, т.е. угол между боковыми вершинами равен $\pi$, и вершина большей валентности имеет и больший модуль. Пусть теперь $N=3, k, l, m, k<l<m$, валентности боковых вершин и $a, b, 1$ - их координаты. Этот тип содержит два дерева, взаимно симметричных относительно оси $O X$. Имеем

$$
a=\frac{-k m+i \sqrt{k l m(k+l+m)}}{m(l+m)}, \quad b=\frac{-l m-i \sqrt{k l m(k+l+m)}}{m(k+m)} .
$$


Отсюда следует, что $|a|^{2}=k(k+l) / m(l+m),|b|^{2}=l(l+m) / m(k+m),|a|<$ $|b|<1$. Угол между соседними боковыми вершинами больше $\pi / 2$, а $|a|$ и $|b|$ монотонно возрастают с возрастанием $k$ и $l$ соответственно.

Наша цель - доказать аналогичные утверждения в случае $N=4$. Пусть $k$, $l, m, n$ - валентности боковых вершин и $x, y, z, 1$ - их координаты. Решая систему (1) относительно $1 / z$ (система содержит три уравнения; исключаем $1 / x$ из 1-го и 2-го и из 1-го и 3 -го и получаем два уравнения, из которых исключаем $1 / y)$, получаем уравнение $F(z, k, l, m, n)=0$ шестой степени по $z$, все коэффициенты которого положительны (к сожалению, громоздкость многочлена $F$ - этот многочлен есть сумма примерно 100 слагаемых — не позволяет его здесь привести). Этот факт используется при доказательстве следующего утверждения.

ПРЕДЛОЖЕНИЕ 1. Если валентность иентра равна 4, то угол между соседними боковыми вершинами больше $\pi / 6$.

ДокАЗАТЕЛЬСтво. Если угол между $m$-вершиной и $n$-вершиной не превосходит $\pi / 6$, то $F$ имеет корень $z_{0}$, такой, что $0 \leqslant \arg \left(z_{0}\right) \leqslant \pi / 6$. Тогда числа $z^{j}$, $j=0, \ldots, 6$, лежат в верхней полуплоскости. Значит их сумма с положительными коэффициентами не может равняться нулю.

ПРеДЛОЖЕНИЕ 2 . Пусть $m \leqslant n$. Тогда

(a) $|z|<1,25$

(b) если $k \leqslant l$ и $n / m \geqslant l / k$ (в частности, если $m \leqslant k \leqslant l \leqslant n$ ), то $|z|<1$;

(c) если $m=n$, mo $|z|>0,5$;

(d) если $2 m=n$, то $0,3<|z|<1$.

ЗАмечАниЕ. $|z|$ может быть больше 1 , даже если $k, l, m<n$. Например, это верно для дерева с циклическим порядком боковых вершин $k=1, l=8, m=9$, $n=10$.

ДОКАЗАТЕЛЬСТВо ПРЕДЛОЖЕНИЯ 2. НаПомним, что многочЛен с вещественными коэффициентами называется устойчивым, если все его корни лежат в левой полуплоскости. Рассмотрим утверждение (а). Нам нужно доказать, что корни многочлена $F$ лежат в круге $|z|<1,25$. При помощи замены переменной $z=(5+5 w) /(4-4 w)$ задачу можно свести к проверке устойчивости некоторого многочлена $G(w, k, l, m, n)=\sum_{j=0}^{6} a_{j} w^{j}$. В соответствии с критерием ЛьенараШипара [3] для этого достаточно доказать положительность коэффициентов многочлена $G$ и положительность определителей

$$
\Delta_{3}=\left|\begin{array}{ccc}
a_{5} & a_{3} & a_{1} \\
a_{6} & a_{4} & a_{2} \\
0 & a_{5} & a_{3}
\end{array}\right|, \quad \Delta_{5}=\left|\begin{array}{ccccc}
a_{5} & a_{3} & a_{1} & 0 & 0 \\
a_{6} & a_{4} & a_{2} & a_{0} & 0 \\
0 & a_{5} & a_{3} & a_{1} & 0 \\
0 & a_{6} & a_{4} & a_{2} & a_{0} \\
0 & 0 & a_{5} & a_{3} & a_{0}
\end{array}\right| .
$$

Здесь $a_{0}, \ldots, a_{6}, \Delta_{3}, \Delta_{5}$ являются многочленами от $k, l, m, n$. Пусть $p(k, l, m, n)$ $=\sum_{i, j} p_{i, j}(m, n) k^{i} l^{j}-$ один из этих многочленов. Достаточно доказать положительность всех $p_{i, j}(m, n)$ при $m \leqslant n$, что является несложной, но утомительной проверкой.

Утверждения (b), (c) и (d) доказываются аналогично. Однако в случае (b) многочлены $p_{i, j}(m, n)$ могут быть неположительны. Мы докажем положитель- 
ность многочленов $q_{s}(k, l, m, n)=\sum_{i+j=s} p_{i, j}(m, n) k^{i} l^{j}$. Многочлены $p_{i, j}$, входящие в $q_{s}$, однородны по $m, n$ и имеют одну и ту же степень. Переходя к новым переменным $\alpha=n / m$ и $\beta=l / k$, мы получаем многочлен от $\beta$, коэффициенты которого являются многочленами от $\alpha$. Для доказательства положительности этого многочлена осталось воспользоваться его возвратностью и тем фактом, что $\alpha \geqslant \beta$.

Численные эксперименты показывают, что при возрастании $m$ модуль $z$ также возрастает и что при возрастании $n$ возрастает угол между $n$-вершиной и соседней с ней. Строгое доказательство этого утверждения удается провести только в случае $k=l$ (схему доказательства, изложенную ниже, не удается провести в общем случае даже при использовании хорошей техники - мощности не хватает). Пусть $l=k$. Тогда $F$ является полным квадратом, $F=G^{2}$, где

$$
G=n(k+n)(2 k+n) z^{3}+3 m n(k+n) z^{2}+3 m n(k+m) z+m(k+m)(2 k+m) .
$$

ПРЕДЛОЖЕНИЕ 3. Пусть $k=l$. Тогда

(a) $|z|$ возрастает с возрастанием $m$;

(b) угол между $n$-вершиной и соседней $m$-вершиной возрастает с возрастанием $n$.

ДокАЗАТЕЛЬСтво. (а) По соображениям непрерывности достаточно доказать, что $\left|z^{2}\right|_{m}^{\prime} \neq 0$ или что $\operatorname{Re}\left(G_{m}^{\prime} \bar{G}_{\bar{z}}^{\prime} \bar{z}\right) \neq 0$. Полагая $z=x+i y$, рассматриваем систему уравнений $\operatorname{Re}(G)=\operatorname{Im}(G)=\operatorname{Re}\left(G_{m}^{\prime} \bar{G}_{\bar{z}}^{\prime} \bar{z}\right)=0$. Исключая из этой системы сначала $y$, а потом $x$, получаем многочлен от $k, m, n$, все коэффициенты которого положительны.

(b) Достаточно доказать, что $z_{n}^{\prime} \neq t z$, где $t \in \mathbb{R}$, или что $\operatorname{Im}\left(z_{n}^{\prime} / z\right) \neq 0$. Далее рассуждаем, как в (а).

Для случая центральной валентности 5 известны только результаты численных экспериментов, так как вычислить соответствующий многочлен 24-й степени (и от пяти параметров $k_{1}, k_{2}, k_{3}, k_{4}, k_{5}$ ) не удается. Известно, однако, что он не обладает свойством положительности коэффициентов. Например, это не так для типа $\langle 10000,1000,50,5,1\rangle$ (вершина валентности 1000 находится в 1, система решается относительно координаты $u$ вершины валентности 1). Поэтому распространить на этот случай утверждение о минимальном угле не удается. Более того, вычисления указывают, что угол между соседними вершинами может быть сколь угодно мал. Модули боковых вершин обладают свойствами, аналогичными свойствам модулей в случае центральной валентности 4 (напомним, что это результаты численных экспериментов). Если $k, l, m, n, p-$ валентности боковых вершин, а $x, y, z, u, 1$ - их координаты, то (а) если $n \leqslant p$, то $|u|<1,4$; (b) если $n=p$, то $|u|>0,7$; (c) если $p=2 n$, то $0,5<|u|<1,1$.

Автор благодарен Герхарду Посту за поддержку и помощь в вычислениях.

\section{ЛИТЕРАТУРА}

1. Shabat G., Zvonkin A. Contemp. Math., 178, 233-275 (1994). 2. Betrema J., Pere D., Zvonkin A. Plane Trees and their Shabat Polynomials. Catalog, LABRI-Universite Bordeaux 92-75, Bordeaux, 1992. 3. Постников M. М. Устойчивые многочлены. Наука, M., 1981. 